Probability, Networks and Algorithms 
Centrum voor Wiskunde en Informatica (CWI) is the national research institute for Mathematics and Computer Science. It is sponsored by the Netherlands Organisation for Scientific Research (NWO).

CWI is a founding member of ERCIM, the European Research Consortium for Informatics and Mathematics.

CWI's research has a theme-oriented structure and is grouped into four clusters. Listed below are the names of the clusters and in parentheses their acronyms.

\section{Probability, Networks and Algorithms (PNA)}

Software Engineering (SEN)

Modelling, Analysis and Simulation (MAS)

Information Systems (INS)

Copyright (C) 2006, Stichting Centrum voor Wiskunde en Informatica

P.O. Box 94079, 1090 GB Amsterdam (NL)

Kruislaan 413, 1098 SJ Amsterdam (NL)

Telephone +31205929333

Telefax +31205924199

ISSN 1386-3711 


\title{
Perfect simulation for length-interacting polygonal Markov fields in the plane
}

\begin{abstract}
The purpose of this paper is to construct perfect samplers for length-interacting Arak-Clifford-Surgailis polygonal Markov fields in the plane [AS1]. This is achieved by providing for the polygonal fields a hard-core-interacting marked point process representation with individual points carrying polygonal loops as their marks. This places us in the general framework of [LieshoutStoica] thus allowing us to adopt the general coupling-from-the-past and clan of ancestors routines for our particular purposes.
\end{abstract}

2000 Mathematics Subject Classification: 60D05, 60G55.

Keywords and Phrases: Arak process, length-interacting polygonal Markov field, perfect sampling, coupling from the past, clan of ancestors algorithm.

Note: This work was carried out under the MUSCLE project. 



\title{
Perfect simulation for length-interacting polygonal Markov fields in the plane
}

\author{
M.N.M. van Lieshout ${ }^{1}$ and T. Schreiber ${ }^{2}$ \\ ${ }^{1} C W I$ \\ P.O. Box 94079, 1090 GB Amsterdam, The Netherlands \\ 2 Faculty of Mathematics 83 Computer Science, Nicolaus Copernicus University \\ ul. Chopina 12-18, 87-100 Toruń, Poland
}

\begin{abstract}
The purpose of this paper is to construct perfect samplers for length-interacting ArakClifford-Surgailis polygonal Markov fields in the plane [2]. This is achieved by providing for the polygonal fields a hard-core-interacting marked point process representation with individual points carrying polygonal loops as their marks. This places us in the general framework of [14] thus allowing us to adopt the general coupling-from-the-past and clan of ancestors routines for our particular purposes.
\end{abstract}

2000 Mathematics Subject Classification: 60D05, 60G55.

Keywords and Phrases: Arak process, length-interacting polygonal Markov field, perfect sampling, coupling from the past, clan of ancestors algorithm.

\section{INTRODUCTION}

Following the ground breaking paper by Propp and Wilson [17], which presented exact samplers for a range of discrete probability distributions including the Ising model at critical temperature, a variety of exact or perfect simulation methods has been developed for absolutely continuous probability distributions. In this paper, we are particularly interested in such methods for polygonal Markov fields observed in planar windows [2].

Our main interest for developing these algorithms is motivated by the fact that the polygonal Markov fields seem to constitute a natural and promising prior for image segmentation purposes. This has first been noted by Clifford and Middleton [5] whereas the first sampler has been developed by Clifford and Nicholls [6]. Even though the original sampler was rather slow, it has been recently re-worked and applied for real data by Paskin and Thrun [16]. A completely different algorithm based on the notion of so-called disagreement loops has recently been developed by Kluszczyński, Van Lieshout and Schreiber [11] and successfully applied for image segmentation [12].

All the above mentioned samplers were, however, based on the classical Metropolis-Hastings and Gibbs-sampling schemes, and the research was mainly concentrated on elaborating new 
efficient moves whereas the corresponding rates of convergence remain unknown. In this context, it is important to develop tools allowing for perfect simulation of the polygonal Markov fields. The present paper deals only with the low-temperature regime for lengthinteracting fields, yet we plan to extend the applicability of the perfect scheme to the hightemperature and area-interacting regimes as well.

The idea underlying the perfect sampling algorith presented in this article is to represent the polygonal field as a marked point process, with individual points carrying polygonal loops as their marks. The interaction in this representation turns out to be repulsive and based on a hard-core inter-contours exclusion rule.

The plan of this paper is as follows. In Section 2.1, we review the construction of the length-interacting colourless Arak process with empty boundary condition, then present a survey of marked point and object processes in Section 2.2. In Section3, we re-formulate the length-interacting Arak process as a hard object process, and derive the mark distribution. The perfect samplers are discussed in the subsequent Section 4 . We summarise our results in Section 5. For the reader's convenience, the paper is concluded by an appendix presenting the so-called dynamic construction for polygonal Markov fields which, even though not directly used in this paper, provides a lot of intuition as to what the polygonal fields are, thus complementing the formal definition provided in Section 2 below.

\section{Polygonal Markov fields and Markov object processes \\ 2.1 Length-interacting polygonal Markov fields}

Let $D \subseteq \mathbb{R}^{2}$ be a bounded open set of strictly positive Lebesgue measure with piecewise smooth boundary $\partial D$, to remain fixed throughout this paper. Define the family $\Gamma_{D}$ of admissible polygonal configurations in $D$ to consist of all planar graphs $\gamma$ in $D$ such that

(P1) $\gamma \cap \partial D=\emptyset$

(P2) all the vertices of $\gamma$ are of degree 2,

(P3) the edges of $\gamma$ do not intersect,

(P4) no two edges of $\gamma$ are co-linear.

In words, $\gamma$ consists of a finite number of disjoint polygons fully contained in $D$ and possibly nested.

For a finite collection $(l)=\left\{l_{i}\right\}_{i=1}^{n}$ of straight lines $l_{i}$ intersecting $D$, denote by $\Gamma_{D}(l)$ the family of $\gamma \in \Gamma_{D}$ that use $(l)$ as their skeleton in the sense that $\gamma \subseteq \bigcup_{i=1}^{n} l_{i}$ and $\gamma \cap l_{i}$ is a single interval of a strictly positive length for each $l_{i}, i=1, \ldots, n$, possibly with some isolated points added. 
Let $\Lambda_{D}$ be the restriction to $D$ of a Poisson line process $\Lambda$ with intensity measure given by the standard isometry-invariant Lebesgue measure $\mu$ on the space of straight lines in $\mathbb{R}^{2}$. Then, the basic polygonal Arak process $\mathcal{A}_{D}$ on $D$ is defined by

$$
\mathbb{P}\left(\mathcal{A}_{D} \in G\right)=\frac{\mathbb{E} \sum_{\gamma \in \Gamma\left(\Lambda_{D}\right) \cap G} \exp (-2 \ell(\gamma))}{\mathbb{E} \sum_{\gamma \in \Gamma\left(\Lambda_{D}\right)} \exp (-2 \ell(\gamma))}
$$

for all $G \subseteq \Gamma_{D}$ that are Borel measurable with respect to the Hausdorff distance topology. Finally, for any reciprocal temperature $\beta>0$, the length-interacting Arak process $\mathcal{A}_{D}^{[\beta]}$ in $D$ is determined in distribution by

$$
\frac{d \mathcal{L}\left(\mathcal{A}_{D}^{[\beta]}\right)}{d \mathcal{L}\left(\mathcal{A}_{D}\right)}[\gamma]:=\frac{\exp (-\beta \ell(\gamma))}{\mathbb{E} \exp \left(-\beta \ell\left(\mathcal{A}_{D}\right)\right)}
$$

with $\mathcal{L}(\cdot)$ standing for the law of the argument random object. The reader is referred to $[2,4]$ for further details.

Note that in the literature on consistent polygonal fields one usually considers free rather than empty boundary conditions, yet the empty boundary object is better suited for our further purposes.

\subsection{Marked point processes}

Let $M$ be a complete separable metric space and take $D$ as above. A planar marked point process $\mathcal{M}_{\mathcal{D}}$ with positions in $D$ and marks in $M$ is a point process on $D \times M$ such that the process of unmarked points is (locally) finite [7]. In other words, realisations of $Y$ are of the form $(y)=\left\{y_{1}=\left(x_{1}, m_{1}\right), \ldots y_{n}=\left(x_{n}, m_{n}\right)\right\}$ where $n \in \mathbb{N}_{0}, x_{i} \in D$, and $m_{i} \in M$ for all $i=1, \ldots, n$, with $x_{i} \neq x_{j}$ for $i \neq j$.

Let $\nu_{M}$ be a probability measure on the Borel $\sigma$-algebra $\mathcal{B}(M)$. We shall restrict attention to marked point processes that are absolutely continuous with respect to the distribution of a unit rate Poisson process $\mathcal{P}_{\mathcal{D}}$ on $D$ marked independently and identically according to $\nu_{M}$.

The Papangelou conditional intensity of a marked point process $\mathcal{M}$ at $(x, m) \in(D \times M) \backslash(y)$ is defined as

$$
\lambda\left((x, m) ;\left\{\left(x_{i}, m_{i}\right)\right\}_{i=1}^{n}\right):=\frac{\left.\frac{d \mathcal{L}(\mathcal{M})}{d \mathcal{L}\left(\mathcal{P}_{\mathcal{D}}\right)}\left[\left\{\left(x_{i}, m_{i}\right)\right\}_{i=1}^{n}\right\} \cup\{(x, m)\}\right]}{\frac{d \mathcal{L}(\mathcal{M})}{d \mathcal{L}\left(\mathcal{P}_{\mathcal{D}}\right)}\left[\left\{\left(x_{i}, m_{i}\right)\right\}_{i=1}^{n}\right]}
$$

whenever $\frac{d \mathcal{L}(\mathcal{M})}{d \mathcal{L}\left(\mathcal{P}_{\mathcal{D}}\right)}\left[\left\{\left(x_{i}, m_{i}\right)\right\}_{i=1}^{n}\right]>0$, and arbitrarily (say 0) otherwise. In other words, $(2.3)$ is the conditional probability of finding a point at $d x$ with mark $d \nu_{M}(m)$ conditional on the configuration elsewhere being $\left\{\left(x_{i}, m_{i}\right)\right\}_{i=1}^{n}$.

Henceforth, we shall assume the following properties to hold:

(M1) $\frac{d \mathcal{L}(\mathcal{M})}{d \mathcal{L}\left(\mathcal{P}_{\mathcal{D}}\right)}$ is hereditary, that is, if marked point pattern $(y)$ is assigned a strictly positive value, so are its subsets, 
(M2) local stability, that is, the Papangelou conditional intensity is bounded from above by some finite constant $\lambda>0$.

For object processes, the mark $m$ of a point at $x$ is a parametrisation of a geometric, planar object $Z(m)$ translated to the location $x$. Such an object is said to be hard if it cannot overlap other objects. In that case,

$$
\lambda\left((x, m) ;\left\{\left(x_{i}, m_{i}\right)\right\}_{i=1}^{n}\right)=0
$$

whenever

$$
x+Z(m) \cap\left[\cup_{i=1}^{n} x_{i}+Z\left(m_{i}\right)\right] \neq \emptyset .
$$

The generic example is to take a constant conditional intensity whenever there is no overlap, which corresponds to the conditional law

$$
\mathcal{L}(\mathcal{M})=\mathcal{L}\left(\bigcup\left\{y \in \mathcal{P}_{\mathcal{D}}\right\} \mid \forall(x, m),\left(x^{\prime}, m^{\prime}\right) \in \mathcal{P}: x \neq x^{\prime} \Rightarrow x+Z(m) \cap x^{\prime}+Z\left(m^{\prime}\right)=\emptyset\right)
$$

of a Poisson process given there is no overlap between the objects in it. Clearly, the map $m \rightarrow Z(m)$ has to be such that the conditioning event is measurable. Note that $\mathcal{M}$ is Markov with respect to the overlapping object relation.

A marked point process is said to be repulsive if $\lambda(\cdot ; \cdot)$ is decreasing in its second argument with respect to set inclusion, attractive if it is increasing. Indeed, in case of repulsion, the more marked points there are, the harder it is to introduce yet another one, and the smaller the conditional intensity.

\section{MARKED POINT PROCESS REPRESENTATION FOR POLYGONAL MARKOV FIELDS}

In this section, we shall show that the length-interacting Arak process is a Markov hard object process (2.4). To do so we recall that, as argued in Schreiber [19] [Section 2.2], the polygonal field $\mathcal{A}_{D}^{[\beta]}$ admits a so-called polymer representation on the space of closed contours in $D$.

Let $\mathcal{C}_{D}$ be the set of all closed polygonal contours in $D$ which do not touch the boundary $\partial D$. For a given finite configuration $(l)=\left\{l_{1}, \ldots, l_{n}\right\}$ of straight lines intersecting $D$ denote by $\mathcal{C}_{D}(l)$ the family of those polygonal contours in $\mathcal{C}_{D}$ which belong to $\Gamma_{D}(l)$. Equip the space $\mathcal{C}_{D}$ with the Hausdorff metric. It is well-known that the Hausdorff metric space on the family of compact subsets of $D \cup \partial D$ is itself compact and hence complete and separable, see e.g. Proposition 1-4-4 in Matheron [15]. The subspace $\mathcal{C}_{D}$ is also a metric space and inherits separability. It is easily seen not to be complete, yet it is a subspace of the compact 
space considered above and hence any point process with marks in $\mathcal{C}_{D}$ falls into the general framework discussed in Subsection 2.2.

We define the so-called free contour measure $\Theta_{D}$ on $\mathcal{C}_{D}$ by putting for $C \subseteq \mathcal{C}_{D}$ measurable with respect to the Borel $\sigma$-field generated by the Hausdorff distance topology,

$$
\Theta_{D}(C)=\int_{\operatorname{Fin}(L[D])} \sum_{\theta \in C \cap \mathcal{C}_{D}(l)} \exp (-2 \ell(\theta)) d \mu^{*}((l))
$$

with $\operatorname{Fin}(L[D])$ standing for the for the family of finite line configurations intersecting $D$ and where $\mu^{*}$ is the measure on $\operatorname{Fin}(L[D])$ given by $d \mu^{*}\left(l_{1}, \ldots, l_{n}\right):=\prod_{i=1}^{n} d \mu\left(l_{i}\right)$ with $\mu$ defined in the discussion preceding (2.1).

For $\beta \in \mathbb{R}$, define the exponential modification $\Theta_{D}^{[\beta]}$ of the free measure $\Theta_{D}$ by

$$
\Theta_{D}^{[\beta]}(d \theta):=\exp (-\beta \ell(\theta)) \Theta_{D}(d \theta)
$$

and let $\mathcal{P}_{\Theta_{D}^{[\beta]}}$ be the Poisson point process on $\mathcal{C}_{D}$ with intensity measure $\Theta_{D}^{[\beta]}$. Then, by (3.1) and (2.1), the polygonal field $\mathcal{A}_{D}^{[\beta]}$ coincides in distribution with the union of contours in $\mathcal{P}_{\Theta_{D}^{[\beta]}}$ conditioned on the event that they are disjoint i.e.

$$
\mathcal{L}\left(\mathcal{A}_{D}^{[\beta]}\right)=\mathcal{L}\left(\bigcup_{\theta \in \mathcal{P}_{\Theta}^{[\beta]}} \theta \mid \forall_{\theta, \theta^{\prime} \in \mathcal{P}_{\Theta_{D}^{[\beta]}}^{[\beta]}} \theta \neq \theta^{\prime} \Rightarrow \theta \cap \theta^{\prime}=\emptyset\right)
$$

see Section 2.2 in [19]. Note that the conditioning in (3.3) makes sense because $\Theta_{D}^{[\beta]}\left(\mathcal{C}_{D}\right)$ is finite as shown in of [19, Section 2.2]. Furthermore, for all bounded open sets $D$ with piecewise smooth boundary, the free contour measures $\Theta_{D}$ as defined in (3.1) arise as the respective restrictions to $\mathcal{C}_{D}$ of the same measure $\Theta$ on $\mathcal{C}:=\bigcup_{n=1}^{\infty} \mathcal{C}_{(-n, n)^{2}}$, in the sequel referred to as the infinite volume free contour measure. In the same way we construct the infinite volume Gibbs-modified measures $\Theta^{[\beta]}$.

In order to place the polymer representation in the setting of [14], it is convenient to identify a given contour collection $\gamma=\left\{\theta_{1}, \ldots, \theta_{k}\right\}$ arising as a realisation of $\mathcal{A}_{D}^{[\beta]}$, with the collection of points $x_{i}:=\iota\left[\theta_{i}\right], i=1, \ldots, k$, carrying the respective contours as their marks, where $\iota[\cdot]$ is a mapping from $\mathcal{C}_{D}$ to $D$. Even though a number of different natural candidates for $\iota[\cdot]$ could be considered, to be specific in the sequel we shall always take $\iota[\theta]$ to be the extreme left point of the contour $\theta$, minimising the $x$-coordinate, with possible ties broken in an arbitrary measurable way. For formal convenience we regard the marks $\theta_{i}$ attached to the points $x_{i} \in D$ as elements of the common space $\mathcal{C}_{0}:=\{\theta \in \mathcal{C} \mid \iota[\theta]=0\}$ shifted to $x_{i}$. Below, for a point $x \in D$ carrying a mark $\theta \in \mathcal{C}_{0}$, we shall reserve the name of shifted mark for the translate of the contour $\theta$ by the vector $x$. It is also convenient for our further purposes to admit in $\mathcal{C}_{0}$ the empty contour $\emptyset$. 
For each $\beta \geq 2$ we must endow the mark space $M=\mathcal{C}_{0}$ with a probability measure $\nu_{M}$. This can be done by the random walk representation of $[20]$ as follows. Let $\Theta_{*}^{[\beta]}$ be determined by the following construction of a $\mathcal{C}_{0}$-valued $\Theta_{*}^{[\beta]}$-distributed random element $\theta$ :

- Simulate a continuous-time random walk $Z_{t}$ governed by the following dynamics

- put $Z_{0}:=0$ and choose the initial direction uniformly in $(0,2 \pi)$,

- between critical events specified below move in a constant direction with speed 1 ,

- with intensity given by 4 times the covered length element update the movement direction, choosing the angle $\phi \in(0,2 \pi)$ between the old and new direction according to the density $|\sin (\phi)| / 4$,

- Consider a killed modification $\tilde{Z}_{t}^{[\beta-2]}$ of $Z_{t}$ by killing $Z_{t}$

- with constant rate $\beta-2$,

- whenever it hits its past trajectory,

- Draw an infinite loop-closing half-line $l^{*}$ beginning at 0 and forming with the initial segment of $\left(Z_{t}\right)_{t \geq 0}$ an angle $\phi^{*} \in(0,2 \pi)$ distributed according to the density $\left|\sin \phi^{*}\right| / 4$,

- If the random walk $\tilde{Z}_{t}^{[\beta-2]}$ hits the loop closing line $l^{*}$ before being killed, and the self-avoiding contour $\theta_{*}$ created by $l^{*}$ and the trajectory of $\tilde{Z}_{t}^{[\beta-2]}$ up to the moment of hitting $l^{*}$ satisfies $\iota\left[\theta_{*}\right]=0$, then

- with probability $\exp \left(-[\beta+2] \ell\left(e^{*}\right)\right)$ output $\theta:=\theta_{*}$, where $e^{*}$ stands for the segment of the loop closing line $l^{*}$ from 0 to its intersection point with $\tilde{Z}_{t}^{[\beta-2]}$,

- otherwise output $\theta:=\emptyset$.

In all remaining cases put $\theta:=\emptyset$.

The following lemma, close in spirit to Lemma 5.1 in [20], is the main result of this section.

Lemma 1. For $\beta \geq 2$ the polygonal Markov field $\mathcal{A}_{D}^{[\beta]}$ coincides in law with the union of contours carried as shifted marks by the $\mathcal{C}_{0}$-marked point process $\mathcal{Y}^{[\beta]}$ in $D$, determined by Papangelou conditional intensity

$$
\lambda\left((x, \theta) ;\left\{\left(x_{i}, \theta_{i}\right)\right\}_{i=1}^{k}\right):= \begin{cases}8 \pi, & \text { if } x+\theta \cap\left[\bigcup_{i=1}^{k} x_{i}+\theta_{i}\right]=\emptyset, x+\theta \subseteq D \\ 0, & \text { otherwise }\end{cases}
$$

with respect to the product of Lebesgue measure on $D$ and $\Theta_{*}^{[\beta]}$ on $\mathcal{C}_{0}$. 
Proof The directed nature of the random walk trajectories as constructed above requires considering for each contour $\theta$ two oriented instances $\theta^{\rightarrow}$ (clockwise) and $\theta^{\leftarrow}$ (anti-clockwise). In view of the polymer representation (3.3) and taking into account that $\Theta_{D}^{[\beta]}$ arises as the restriction of $\Theta^{[\beta]}$ to $\mathcal{C}_{D}$, by the construction of $\Theta_{*}^{[\beta]}$ the assertion of the lemma will follow as soon as we show that for each $x \in \mathbb{R}^{2}$ and $\theta \in \mathcal{C}_{0}$ we have

$$
\begin{aligned}
& 16 \pi d x \mathrm{e}^{-[\beta+2] \ell\left(e^{*}\right)} \mathbb{P}\left(\tilde{Z}_{t}^{[\beta-2]} \text { reaches } l^{*} \text { and the resulting contour falls into } x+d \theta^{\rightarrow}\right) \\
& =\Theta^{[\beta]}(d \theta),
\end{aligned}
$$

where $e^{*}$ stands for the last segment of $\theta^{\rightarrow}$ counting from $x$ as the initial vertex, which is to coincide with the segment of the loop-closing line $l^{*}$ joining its intersection point with $\tilde{Z}_{t}^{[\beta-2]}$ to 0 . Indeed, the same relation holds then for $\theta^{\leftarrow}$, whence adding versions of (3.5) for $\theta^{\rightarrow}$ and $\theta^{\leftarrow}$, which amounts to taking into account two possible directions in which the random walk can move along $\theta$, will yield $2 \Theta^{[\beta]}(d \theta)$ on the right hand side, whence (3.4) will follow.

To establish (3.5), we observe that the probability element

$$
\mathbb{P}\left(\tilde{Z}_{t}^{[\beta-2]} \text { reaches } l^{*} \text { and the resulting contour falls into } x+d \theta^{\rightarrow}\right)
$$

is exactly

$$
\frac{1}{4[\mu \times \mu]\left(\left\{\left(l, l^{*}\right) \mid l \cap l^{*} \in d x\right\}\right)} \exp \left(-[(\beta-2)+4] \ell\left(\theta \backslash e^{*}\right)\right) \prod_{i=1}^{k} d \mu\left(l\left[e_{k}\right]\right),
$$

where $e_{1}, \ldots, e_{k}$ are all segments of $\theta$ including $e^{*}$, while $l\left[e_{i}\right]$ stands for the straight line determined by $e_{i}$. Indeed,

- the prefactor $\left[4[\mu \times \mu]\left(\left\{\left(l, l^{*}\right) \mid l \cap l^{*} \in d x\right\}\right)\right]^{-1}$ comes from the choice of the lines containing respectively the initial segment of $\theta^{\rightarrow}$ (counting from $x$ ) and $l^{*}$ as well as from the choice between two equiprobable directions on each of these lines,

- for the remaining segments we use the fact that, for any given straight line $l_{0}, \mu(\{l \mid l \cap$ $\left.\left.l_{0} \in d \ell, \angle\left(l, l_{0}\right) \in d \phi\right\}\right)=|\sin \phi| d \ell d \phi$ with $d \ell$ standing for the length element on $l_{0}$ and with $\angle\left(l_{0}, l\right)$ denoting the angle between $l$ and $l_{0}$, see Proposition 3.1 in [2] (as well as the argument justifying the dynamic representation of the Arak process in Section 4 of Arak \& Surgailis [2] and the proof of Lemma 1 in Schreiber [19]). Note that the direction update intensity was set to 4 to coincide with $\int_{0}^{2 \pi}|\sin \phi|=4$.

To get the required relation (3.5) it is now enough to use (3.6), recall the definition of $\Theta^{[\beta]}$ and observe that $[\mu \times \mu]\left(\left\{\left(l, l^{*}\right) \mid l \cap l^{*} \in d x\right\}\right)=4 \pi d x$ as follows by standard integral geometry, cf. [2, Proposition 3.1]. The proof is complete. 


\section{Perfect Simulation USing Spatial Birth-AND-Death Processes}

In this section, we consider two exact sampling methods based on the classic idea to simulate from planar point process models by means of running a spatial birth-and-death process. Coupling from the past techniques (CFTP) for point processes were introduced in [9] for a special model. The generalisation to locally stable point processes can be found in [10], and to marked patterns in [14]; [13] presents an adaptive variation on the theme.

Initialise $T=1$, and let $\mathcal{Y}(0)$ be a realisation of a Poisson process of rate $8 \pi$ in $D$, marked i.i.d. according to $\nu_{M}=\Theta_{*}^{[\beta]}$.

\section{Algorithm 1. (CFTP)}

- Extend $\mathcal{Y}(\cdot)$ backwards to time $-T$ by means of a spatial birth-and-death process with birth rate $8 \pi d x \Theta_{*}^{[\beta]}(d \theta)$ and unit death rate.

- Generate $L^{-T}(\cdot)$ (lower process) and $U^{-T}(\cdot)$ (upper process) forwards in time as follows:

- set $L^{-T}(-T)=\emptyset$ and $U^{-T}(-T)=\mathcal{Y}(-T)$;

- if $\mathcal{Y}(\cdot)$ experiences a backward birth, i.e. $\mathcal{Y}(t-)=\mathcal{Y}(t) \cup\{(x, \theta)\}$ for some $(x, \theta) \notin$ $\mathcal{Y}(t)$, where $\mathcal{Y}(t-)$ denotes the state just prior to time $t$, delete $(x, \theta)$ from $L^{-T}(t-)$ and $U^{-T}(t-)$;

- if $\mathcal{Y}(\cdot)$ experiences a backward death, i.e. $\mathcal{Y}(t-)=\mathcal{Y}(t) \backslash\{(x, \theta)\}$ for some $(x, \theta) \in$ $\mathcal{Y}(t)$, the marked point $(x, \theta)$ is added to $L^{-T}(t-)$ iff

$$
[x+\theta] \cap \bigcup_{\left(x_{i}, \theta_{i}\right) \in U^{-T}(t-)}\left[x_{i}+\theta_{i}\right]=\emptyset
$$

and to $U^{-T}(t-)$ iff

$$
[x+\theta] \cap \bigcup_{\left(x_{i}, \theta_{i}\right) \in L^{-T}(t-)}\left[x_{i}+\theta_{i}\right]=\emptyset .
$$

- If $U^{-T}(0)=L^{-T}(0)$ stop. Else set $T=2 T$ and repeat.

- Return $U^{-T}(0)$.

The clan of ancestors [8] algorithm is similar in flavour. It has the advantage of avoiding the birth of marked points that will have no influence on the final outcome, but does not exploit the repulsive behaviour of the hard core contour process. It tends to be better than coupling from the past for low intensities, worse for higher ones [14].

Let $\mathcal{Y}(0)$ be a realisation of a Poisson process of rate $8 \pi$ in $D$, marked i.i.d. according to $\nu_{M}=\Theta_{*}^{[\beta]}$. Initialise the clan of ancestors as $A=\mathcal{Y}(0)$. 


\section{Algorithm 2. (Clan of Ancestors)}

- Extend $\mathcal{Y}(\cdot)$ backwards by means of a spatial birth-and-death process with birth rate $8 \pi d x \Theta_{*}^{[\beta]}(d \theta)$ and unit death rate. At each (backward) death incident $\mathcal{Y}(t-)=\mathcal{Y}(t) \backslash$ $\{(x, \theta)\}$ for some $t<0$ and $(x, \theta) \in A \cap \mathcal{Y}(t)$, add the marked points $\left(x^{\prime}, \theta^{\prime}\right) \in \mathcal{Y}(t-)$ for which $x^{\prime}+\theta^{\prime} \cap x+\theta \neq \emptyset$ to $A$. The backwards sweep ends when $A_{t}=A \cap \mathcal{Y}(t)=\emptyset$. The stopping time thus obtained is denoted by $-T$.

- Generate $\mathcal{Z}(\cdot)$ forwards in time as follows:

- set $\mathcal{Z}(-T)=\emptyset$;

- if $\mathcal{Y}(\cdot)$ experiences a backward birth, i.e. $\mathcal{Y}(t-)=\mathcal{Y}(t) \cup\{(x, \theta)\}$ for some $(x, \theta) \notin$ $\mathcal{Y}(t)$, delete $(x, \theta)$ from $\mathcal{Z}(t-)$;

- if $\mathcal{Y}(\cdot)$ experiences a backward death, i.e. $\mathcal{Y}(t-)=\mathcal{Y}(t) \backslash\{(x, \theta)\}$ for some $(x, \theta) \in$ $A_{t}$, the marked point $(x, \theta)$ is added to $\mathcal{Z}(t-)$ iff

$$
[x+\theta] \cap \bigcup_{\left(x_{i}, \theta_{i}\right) \in \mathcal{Z}(t-)}\left[x_{i}+\theta_{i}\right]=\emptyset
$$

if $(x, \theta) \notin A_{t}$ then $\mathcal{Z}(t)=\mathcal{Z}(t-)$ remains unchanged.

- Return $\mathcal{Z}(0)$.

Some realisations obtained by Algorithms 1-2 implemented in C++ using the library MPPLIB [21] are presented in Figures 1-2. The execution time is in the order of seconds for Figure 1, minutes for those in Figure 2 under Fedora.

Both Algorithm 1 and 2 can be placed in the general framework discussed in [14] as soon as we are able to verify the conditions (M1) and (M2) for the Papangelou conditional intensity $\lambda(\cdot ; \cdot)$ as given in (3.4), as well as the repulsivity of $\lambda(\cdot ; \cdot)$ as needed for the validity of the Algorithm 1. All these required relations are, however, self-evident, which leads us to the following lemma concluding the current section.

Lemma 2. The polygonal Markov field $\mathcal{A}_{D}^{[\beta]}$ coincides in distribution with both $U^{-T}(0)=$ $L^{-T}(0)$ as constructed in Algorithm 1 and with $\mathcal{Z}(0)$ as output by Algorithm 2.

\section{Conclusion}

In this paper, we designed perfect simulation algorithms for length-interacting colourless Arak polygonal Markov fields observed in planar windows. To do so, we reformulated the model as a hard object process, derived the mark distribution, and specialised the coupling from the past and clan of ancestors algorithms developed for hard object processes to our 

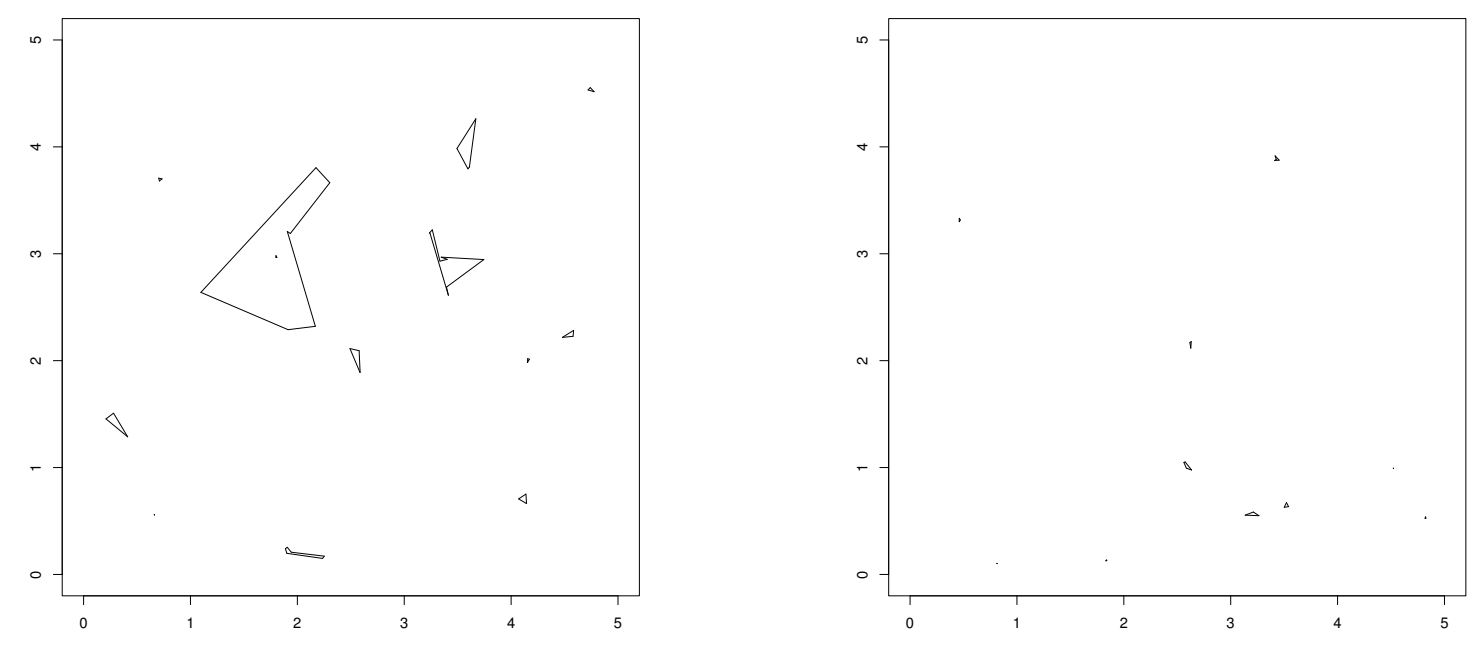

Figure 1: Perfect samples from the length-interacting Arak process $(2.2)$ in $D=[0,5]^{2}$ by Algorithm 2 for $\beta=2$ (left) and $\beta=3$ (right).

particular model. The clan of ancestors method is fastest for low intensities; coupling from the past applies to a wider range of parameter values ( $\beta$ and window size). We aim at constructing high temperature perfect samplers as well, which is the subject of our current work in progress.

An alternative to spatial birth-and-death based simulation is to use the Metropolis-Hastings framework [6]. In contrast to a spatial birth-and-death sampler which accepts all proposed transitions, a Metropolis-Hastings algorithm accepts a new state with a probability that depends on the likelihood ratio of the new state compared to the current one. Note that transitions do not need to be limited to births and deaths; for example, one might wish to alter the angle between two edges, or the position of a vertex. Although such flexibility may be very desirable in practice to improve the mixing time, it also implies that it may be harder to design a perfect version than for the dynamics discusssed in Section 4 . Even in the simplest case with birth and death proposals only, one has to discretise $D$ in order to ensure that deaths are accepted with probability 1 , and be careful which point to delete, so as to maintain the set inclusion order between the upper and lower processes $U$ and $L$ 

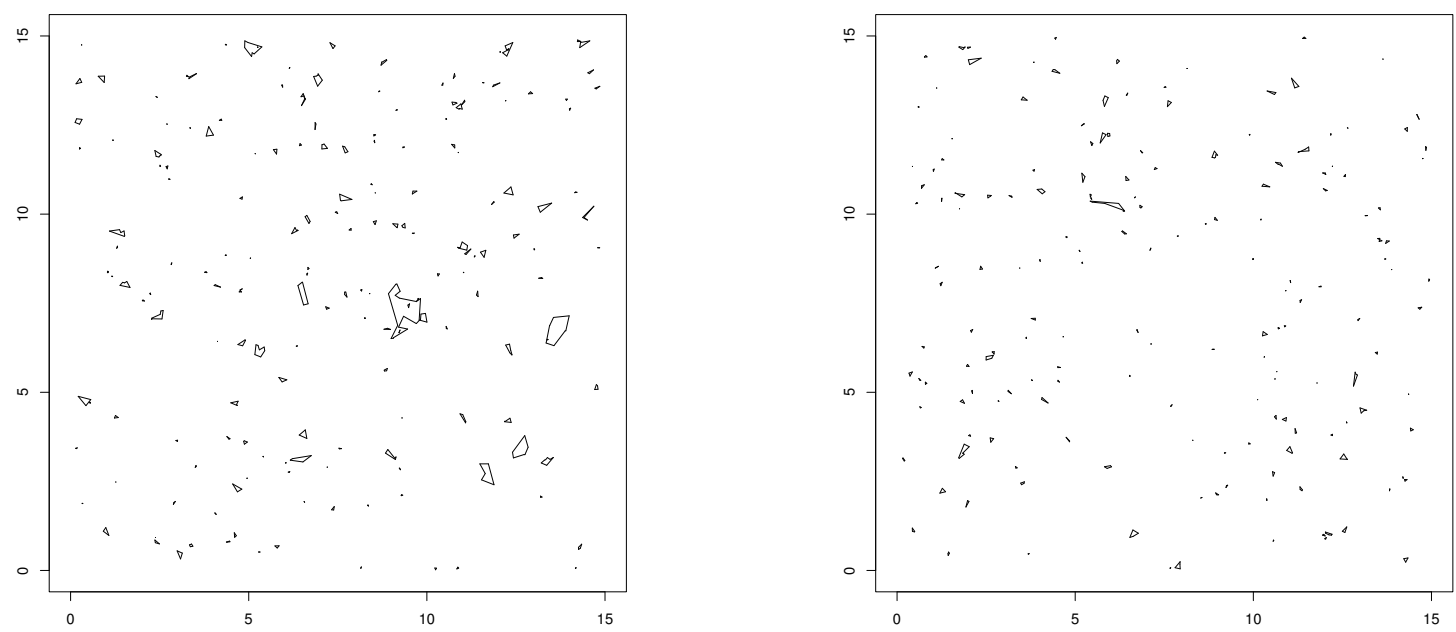

Figure 2: Perfect samples from the length-interacting Arak process $(2.2)$ in $D=[0,15]^{2}$ by Algorithm 1 for $\beta=2$ (left) and $\beta=3$ (right).

(cf. Algorithm 1). Simulation studies [14] suggest the increased complexity of the resulting perfect algorithm is not repaid by increased efficiency, so we do not pursue the topic here. For further details on perfect Metropolis-Hastings sampling, see [10, 14].

\section{Appendix}

Below, we discuss the dynamic representation and some further properties of the basic Arak process, see Arak \& Surgailis [2], Section 4, for the dynamic representation. For a fixed bounded open convex domain $D$ we shall construct the basic Arak process $\mathcal{A}_{D}^{*}$ with free boundary conditions (unlike in (2.1) where empty boundary conditions are imposed).

\subsection{Dynamic construction of the basic Arak process}

We interpret the domain $D$ as a set of time-space points $(t, y) \in D$, with $t$ referred to as the time coordinate and with $y$ standing for the spatial coordinate of a particle at the time $t$. In this language, a straight line segment in $D$ stands for a piece of the time-space trajectory of a freely moving particle. For a straight line $l$ non-parallel to the time axis and crossing the domain $D$ we define in the obvious way its entry point to $D, \operatorname{in}(l, D) \in \partial D$ and its exit point 
$\operatorname{out}(l, D) \in \partial D$.

We choose the time-space birth coordinates for the new particles according to a homogeneous Poisson point process of intensity $\pi$ in $D$ (interior birth sites) superposed with a Poisson point process on the boundary (boundary birth sites) with the intensity measure

$$
\kappa(B)=\mathbb{E} \operatorname{card}\{l \in \Lambda, \operatorname{in}(l, D) \in B\}, B \subseteq \partial D .
$$

Each interior birth site emits two particles, moving with initial velocities $v^{\prime}$ and $v^{\prime \prime}$ chosen according to the joint distribution

$$
\theta\left(d v^{\prime}, d v^{\prime \prime}\right):=\pi^{-1}\left|v^{\prime}-v^{\prime \prime}\right|\left(1+v^{2}\right)^{-3 / 2}\left(1+v^{\prime 2}\right)^{-3 / 2} d v^{\prime} d v^{\prime \prime} .
$$

This can be shown to be equivalent to choosing the directions of the straight lines representing the space-time trajectories of the emitted particles according to the distribution of the typical angle between two lines of $\Lambda$, see Sections 3 and 4 in [2], and the references therein. It is also easily seen that the value of angle $\phi \in(0, \pi)$ between these lines is distributed according to the density $\sin (\phi) / 2$. Each boundary birth site $x \in \partial D$ yields one particle with initial speed $v$ determined according to the distribution $\theta_{x}(d v)$ identified by requiring that the direction of the line entering $D$ at $x$ and representing the time-space trajectory of the emitted particle be chosen according to the distribution of a straight line $l \in \Lambda$ conditioned on the event $\{x=\operatorname{in}(l, D)\}$.

All the particles evolve independently in time according to the following rules.

(E1) Between the critical moments listed below each particle moves freely with constant velocity so that $d y=v d t$,

(E2) When a particle touches the boundary $\partial D$, it dies,

(E3) In case of a collision of two particles (equal spatial coordinates $y$ at some moment $t$ with $(t, y) \in D)$, both of them die,

(E4) The time evolution of the velocity $v_{t}$ of an individual particle is given by a pure-jump Markov process so that

$$
\mathbb{P}\left(v_{t+d t} \in d u \mid v_{t}=v\right)=q(v, d u) d t
$$

for the transition kernel

$$
q(v, d u):=|u-v|\left(1+u^{2}\right)^{-3 / 2} d u d t
$$


It is worth noting that, in full analogy with the discussion following (6.2), the (sharp) angle between the straight lines representing the space-time trajectories of the particle before and after the velocity update is distributed according to the typical angle between two lines of $\Lambda$.

It has been proved (see Lemma 4.1 in [2]) that with the above construction of the interacting particle system, the time-space trajectories traced by the evolving particles coincide in distribution with the Arak process $\mathcal{A}_{D}^{*}$ defined as in (2.1) with the family $\Gamma_{D}$ of admissible polygonal configurations extended to $\Gamma_{D}^{*}$ allowing also for partial contours chopped off by the boundary, which amounts to admitting not only internal vertices of degree 2, as in (P2), but also boundary vertices of degree 1 .

\subsection{Properties of the basic Arak process}

As already mentioned in the introductory section, and as shown in Arak \& Surgailis [2], the basic Arak process $\mathcal{A}_{D}^{*}$ enjoys a number of striking properties. The two-dimensional germ Markov property, stating that the conditional distribution of the field inside a bounded region with piecewise smooth boundary given the outside configuration only depends on the trace of this configuration on the boundary (intersection points and intersection directions) is an immediate consequence of the Gibbsian definition. The next important property is the consistency: for bounded open and convex $D_{1}$ and $D_{2}$ with $D_{1} \subseteq D_{2}$ the restriction of $\mathcal{A}_{D_{2}}^{*}$ to $D_{1}$ coincides in distribution with $\mathcal{A}_{D_{1}}^{*}$, see [2, Theorem 4.1]. This immediately allows us to define the infinite volume Arak process $\mathcal{A}$, which inherits the isometry invariance of the finite volume Gibbsian definition and which is a thermodynamic limit for $\mathcal{A}^{[0]}$. By the results of Schreiber [18], this corresponds to the unique infinite-volume bounded-density stationary evolution of the particle system discussed in Subsection 6.1 above. Interestingly, the intersection of the Arak process $\mathcal{A}$ with any fixed straight line is a Poisson point process of intensity 2, see [2]. Moreover, the partition function for the Arak process can be explicitly evaluated: it is known that

$$
\mathbb{E} \sum_{\delta \in \Gamma_{D}^{*}\left(\Lambda_{D}\right)} \exp (-2 \ell(\delta))=\exp (\pi \operatorname{Area}(D))
$$

see Theorem 4.1 in [2] (note that the prefactor $2 \exp (\ell(\partial D)$ ), present in the quoted theorem, is absent here because we take the law of $\Lambda$ rather than the unnormalised measure $\mu^{*}$ as the reference measure and, moreover, we do not sum over two different admissible black/white colourings of each polygonal configuration). It should be emphasised that these exact results are only available for $\mathcal{A}$ and not for $\mathcal{A}^{[\beta]}, \beta \neq 0$.

Interestingly, there exists a much broader class of consistent polygonal Markov fields admitting analogous dynamic representations, possibly enhanced to allow for vertices of higher degrees (3 and 4), see [2]. The question of characterising the class of all polygonal Markov 
fields admitting dynamic representations is far from being trivial and falls beyond the scope of this article. A conjectured description of this class has been provided in Arak, Clifford \& Surgailis [4], where a very nice alternative point- rather than line-based representation of polygonal fields is also discussed.

Acknowledgements We gratefully acknowledge support from the EC 6th Framework Programme Priority 2 Information Society Technology Network of Excellence MUSCLE (Multimedia Understanding through Semantics, Computation and Learning; FP6-507752). Tomasz Schreiber also wishes to express his gratitude for support from the Polish Minister of Scientific Research and Information Technology grant 1 P03A 01828 (2005-2007) and to warmly thank for hospitality of the Centrum voor Wiskunde en Informatica (CWI), Amsterdam, The Netherlands, where this research was carried out. He also addresses his special thanks to the RDSES programme of the European Science Foundation, which provided numerous sources of inspiration for initiating this research.

\section{REFERENCES}

1. Arak, T. (1982) On Markovian random fields with finite number of values, 4th USSRJapan symposium on probability theory and mathematical statistics, Abstracts of Communications, Tbilisi.

2. Arak, T. And Surgailis, D. (1989) Markov Fields with polygonal realisations, Probab. Th. Rel. Fields 80, 543-579.

3. Arak, T. And Surgailis, D. (1991) Consistent polygonal fields, Probab. Th. Rel. Fields 89, 319-346.

4. Arak, T., Clifford, P., And Surgailis, D. (1993) Point-based polygonal models for random graphs, Adv. Appl. Probab. 25, 348-372.

5. Clifford, P. And Middleton, R.D. (1989) Reconstruction of polygonal images, Journal of Applied Statistics 16, 409-422.

6. Clifford, P. ANd Nicholls, G. (1994) A Metropolis sampler for polygonal image reconstruction, available at:

http://www.stats.ox.ac.uk/ clifford/papers/met_poly.html.

7. Daley, D.J. And Vere-Jones, D. (1988) An Introduction to the theory of point processes, Springer Series in Statistics, Springer-Verlag, New York.

8. Fernández, R., Ferrari, P., and Garcia, N. (2002) Perfect simulation for Perfect simulation for interacting point processes, loss networks and Ising models, Stochastic Process. Appl. 102, 63-88. 
9. Kendall, W.S. (1998) Perfect simulation for the area-interaction point process, In: L. Accardi and C. Heyde (Eds.), Probability towards 2000, Springer, New York, 218234.

10. Kendall, W.S. And Møller, J. (2000) Perfect simulation using dominating processes on ordered spaces, with application to locally stable point processes, Adv. in Appl. Probab. 32, 844-865.

11. Kluszczyński, R., Lieshout, M.N.M. van, And Schreiber, T. (2006) Image segmentation by polygonal Markov fields, to appear in Annals of the Institute of Mathematical Statistics.

12. Kluszczyński, R., Lieshout, M.N.M. van, and Schreiber, T. (2005) An algorithm for binary image segmentation using polygonal Markov fields, In: F. Roli and S. Vitulano (Eds.), Image Analysis and Processing, Proceedings of the 13th International Conference on Image Analysis and Processing, Lecture Notes in Computer Science 3615, 383-390.

13. Lieshout, M.N.M. van and Baddeley, A.J. (2002) Extrapolating and interpolating spatial patterns, In: A.B. Lawson and D.G.T. Denison (Eds.), Spatial cluster modelling, Chapman and Hall/CRC, Boca Raton, 61-86.

14. Lieshout, M.N.M. van And Stoica, R.S. (2006) Perfect simulation for marked point processes, to appear in Computational Statistics and Data Analysis.

15. Matheron, G. (1975) Random sets and integral geometry, Wiley, New York.

16. Paskin, M.A. And Thrun, S. (2005) Robotic mapping with polygonal random fields, In Proceedings in Artificial Intelligence UAI-05.

17. Propp, J.G. And Wilson, D.B. (1996) Exact sampling with coupled Markov chains and applications to statistical mechanics, Random Structures Algorithms 9, 223-252.

18. Schreiber, T. (2004) Mixing properties for polygonal Markov fields in the plane, submitted, available at: http://www.mat.uni.torun.pl/preprints, 18-2003.

19. Schreiber, T. (2005) Random dynamics and thermodynamic limits for polygonal Markov fields in the plane, Adv. Appl. Probab. 37, 884-907.

20. Schreiber, T. (2006) Dobrushin-Kotecký-Schlosman theorem for polygonal Markov fields in the plane, to appear in Journal of Statistical Physics.

21. Steenbeek, A.G., Lieshout, M.N.M. van, and Stoica, R.S. with contributions from P. Gregori and K.K. Berthelsen (2002-2003) MPPBLiB, a C++ library for marked point processes, CWI, Amsterdam. 